\title{
PERFORMANCE ANALYSIS FOR GPRS WITH PRIORITIZED AND NON-PRIORITIZED MOBILITY MANAGEMENT PROCEDURES
}

\author{
Karann Chew, Rahim Tafazolli
}

University of Surrey, United Kingdom

\begin{abstract}
GPRS is part of the evolution path towards 3G. This paper investigates the effect of user mobility on packet transmission in GPRS. A simulator was developed to study the end-to-end behaviour of data transmission between a mobile client and a fixed server via GPRS and Internet. Simulation results show interruption in data delivery when MS executes a routing area update procedure. A fair queuing algorithm is proposed to minimise update delay and packet loss. In addition, we give priority to all the mobility management-related packets to ensure that all MMrelated packets are processed and reach its destination with minimal delay. The obtained simulation results demonstrated that the proposed techniques give better routing area update performance than FIFO service.
\end{abstract}

\section{INTRODUCTION}

The General Packet Radio Service (GPRS) [1] is part of the evolution path towards third generation $(3 \mathrm{G})$ mobile system. It is designed to provide packet-based data service in a cellular system based on GSM. GPRS has its own set of mobility management signalling for different types of routing area updates. During a routing area update operation, the down stream and up stream packet transfer to and from MS is momentarily interrupted. The latency of packet delivery is increased due to these interruptions. In addition, packets could be mis-routed prior to routing area update completion, and this results in packet loss.

The simplest and most popular scheme for servicing packet, First In First Out (FIFO), suffers from the drawback that some traffic might get an unfair share of resource at the expense of other users. In the case of Mobility Management (MM) in GPRS, MAP signalling messages in SGSN and GGSN might not get a fair share of resource. This is because the volume of LLC and GTP packets, carrying both data and signalling packets, outnumbers MAP packets. To counter the unfairness, we propose a Fair Queuing algorithm that keeps separate queues for different types of packets. The queues are served in a round-robin manner, this ensures that MAP signalling messages get a fair share of resource as compared to LLC and GTP. In addition, to further speed up the routing area update procedures, we give priority to all the MM-related LLC and GTP packets. This ensures that all MM-related packets are processed and reach its destination with minimal delay.
This paper first investigates the effects of user mobility on packet transmission in GPRS. A simulator was developed to study the end-to-end data transmission between a mobile client and a fixed Internet server connected via Internet and a GPRS network. The complete architecture of the GPRS was modelled. The analysis focuses on mobility management functionalities and data packet transmission, particularly in the IP layer. Simulation results are presented, showing interruption in data transmission in term of routing area update completion time, packet loss, file download time and ftp throughput when MS execute different types of Routing Area Update procedures. Furthermore, techniques based on service scheduling are implemented to improve the handoff performance in GRPS. We compared the disruption due to routing area updates under three queuing algorithms: FIFO, Fair Queuing with Round Robin, and Priority Queuing for mobility managementrelated messages. Simulation results obtained show that the proposed techniques demonstrate better handoff performance, the best being Priority Queuing, followed by Fair Queuing and finally FIFO.

Following this introduction, Section II presents a description on GPRS, particularly the principle of mobility management in GPRS. The schemes to improve mobility management performance are described $\mathrm{n}$ Section III. In Section IV, the simulation methodology and the simulation environment is presented. Performance evaluation, analysis and discussion are also done in Section IV. Section V concludes this paper.

\section{MOBILITY MANAGEMENT IN GPRS}

\section{Network Architecture}

GPRS is a packet-switched data service for the Global System for Mobile Communications (GSM) standardized by European Telecommunications Standards Institute (ETSI). It introduces a totally new backbone network based on IP. Two new network elements, namely Serving GPRS Support Node (SGSN) and Gateway GPRS Support Node (GGSN) are added in GSM architecture. SGSN and GGSN are mobile data routers they are interconnected via an IP backbone network. SGSN handles packet data traffic of subscribers that are logically located within the SGSN service area. GGSN provides interface to external IP 
networks such as the Internet, enterprise intranets or other GPRS networks,

\section{Mobility Management}

Since GPRS is a cellular network, its mobility management functions are, in principle, the same as that of GSM. As a MS moves from one Routing Area (which is of smaller size that Location Area) to another, database for mobility management are updated accordingly in SGSN, GGSN, and/or HLR to track its location. Location management consists of two levels: GGSN and HLR track MS up to it serving SGSN; whereas SGSN keep track of MS at the routing area or cell level depending on mobility management state of MN.

Routeing Area and Cell Updates. Three different types of updates are possible in GPRS mobility management:

1. Inter-SGSN routing area update (RAU). This is executed when MS moved into a new routing area (RA) administered by a SGSN different from that of the old RA. The new SGSN realizes that the MS has changed to its area and requests the old SGSN to send the PDP contexts of the user. The new SGSN later informs the old SGSN about the user's new routing context. In addition, the HLR, GGSN and, if needed, the MSC/VLR are informed about the user's new SGSN.

2. Intra-SGSN routing area update. The MS has moved to an RA attached to the same SGSN as the old RA. In this case, the affected SGSN has already had the necessary user profile. Since the routing context does not change, there is no need to inform other network elements, such as GGSN or HLR.

3. Cell updates. This applies only to MS that is in active state and has moved to a cell that belongs to the same RA.

Inter-SGSN routing update procedure, as shown in Figure 1, is the most complicated procedure among three types of updates described above. As MS changes from one SGSN area to another, and it must establish a new logical link context between with the new SGSN, new SGSN, in turn, informs GGSN and HLR about the new location of the MS.

\section{Effect of Changing Routing Area in GPRS}

During a routing area update, MS momentarily halt its packet transmission and reception. The packet delivery process resumes after completing the routing area update procedure i.e. after MS receiving the GMM Routing Area Update Accept message from the new SGSN and after new SGSN receiving GMM Routing Area Update Request Complete message from MS (see Figure 1). This results in delayed packet deliveries, affecting different applications in different ways. In the case of realtime interactive application, if packets arrive after playout delay, the packets are dropped. For non- realtime TCP-based application, the TCP might view such losses as a congestion in network and trigger recovery procedures which reduce its throughput.

As a MS changes its routing area whilst in an active session, packets already in the old routing path as well as future packets need to be routed to the new cell. A new route, through which new packets will be delivered, is established during the routing-area update procedures. However, packets could be mis-routed to the old route if routing area update procedure is not accomplished in a timely manner. There are different types of packet loss due to re-routing delays, they includes LLC packets between old BSC and new BSC; GTP packets between old SGSN and new SGSN. Hence, in addition to delayed packet deliveries, service quality is also affected by packet loss during handoff.

These ill effects are more prominent for the case of inter-SGSN routing area update, where at least 14 messages are involved, among MS, new SGSN, old SGSN, GGSN, HLR (this does not including securityrelated messages, and is for the case of non-combined RA/LA update).

\section{QUEUING ALGORITHM TO IMPROVE MOBILITY MANAGEMENT}

The ill effects due to routing area updates can be minimized by reducing the completion time of routing area update procedure. This can be achieved by implementing queuing and scheduling algorithms in certain network nodes such as SGSN and GGSN. Queuing algorithms [2] deal with promptness of packet delivery, bandwidth and buffer space allocation. Various schemes could be implemented to provide quality of service in a packet-based network. The simplest and most popular scheme, first in first out (FIFO) suffers from the drawback that some traffic might get an unfair share of resource at the expense of others. In the case of GPRS, MAP signalling messages might not get a fair share of resource if GSNs are serving using FIFO scheme. This is because the volume of LLC and GTP packets, carrying both data and signalling packets, is much larger than that of MAP packets.

To counter the unfairness of FIFO, we introduce fair queuing (FQ) in servicing the packets. The important feature of FQ is fair allocation of bandwidth among various users and the ability to control delay in delivery of packet. FQ protects the well-behaved users from the ill-behaved ones. In this case, the well-behaved users are the MAP packets while the ill-behaved ones are GTP and LLC packets. We propose a FQ algorithm that keeps separate queues for different types of packets to be incorporated in SGSNs and GGSN. These queues are served in a round-robin manner. This ensures that MAP signalling messages get a fair share of resource as 
compared to LLC and GTP packets which carry both data and signalling packets.

In addition, to further speed up the routing area update procedures, priorities are given to all the MM-related LLC and GTP packets. This ensures that all MMrelated packets are processed and reach its destination with minimal delay. This in turn reduces the total time for completing a routing area update procedures, and hence minimising the disruption on data delivery.

Our model and simulation compared the performance of packet delivery in GPRS under the effect of user mobility. We also compared the disruption of routing area update under three queuing algorithms described above, namely FIFO, Fair Queuing and Priority Queuing. Our results demonstrated that the proposed schemes give better performance than of FIFO.

\section{SIMULATION}

\section{Simulation Structure}

The simulator was implemented using OPNET [3]. A complete architecture of the GPRS, as shown in Figure 2, was modelled - from MSs to the application servers. Our implementation investigated the end-to-end performance at network (IP) layers and above. Figure 2 shows a 'trimmed down' version of the simulator, the actual model consists of 1 HLR, 1 GGSN, 3 SGSNs, 9 BSCs, 30 BTSs and hundreds of MSs. All nodes are hierarchically decomposed into different modules and subsequently into state transition diagrams.

The analysis focuses on data packet transmission and mobility management functionalities. It investigates the effect of mobility in packet delivery in GPRS. Various mobility management functions such as GPRS Attach, Rouging Area Update, Cell Update, Periodical Routing Area Update, Paging and PDP Context Activation have been implemented.

The traffic model consists of two parts - self-similar traffic and TCP-based FTP and HTTP traffic. IP packet source modules are used to create fractal Internet traffic [4], for both downlink and uplink traffic. The network load was dictated by the amount of traffic produced by this source. In addition to the self-similar traffic, FTP and HTTP traffic were also generated in client-server models. These were used to monitor how the TCP based application is influenced by mobility. Each MS is associated with a traffic source and sink module, while a number of selected MSs run FTP or HTTP. All traffic passes through BSS, SGSN, GGSN and the Internet cloud. Some mobility management related messages are also transferred between SGSNs and HLR via a SS7 module.
We assume here that the time spent in a cell is exponentially distributed with mean proportional to the cell radius and inverse proportional to the average speed. After spending some time in one cell, MS moves into either its current cell or any of its six adjacent cells with equal probability. MS obtains a cell ID after each move and subsequently performs either a cell update, intra-SGSN routing area update or interSGSN routing area update.

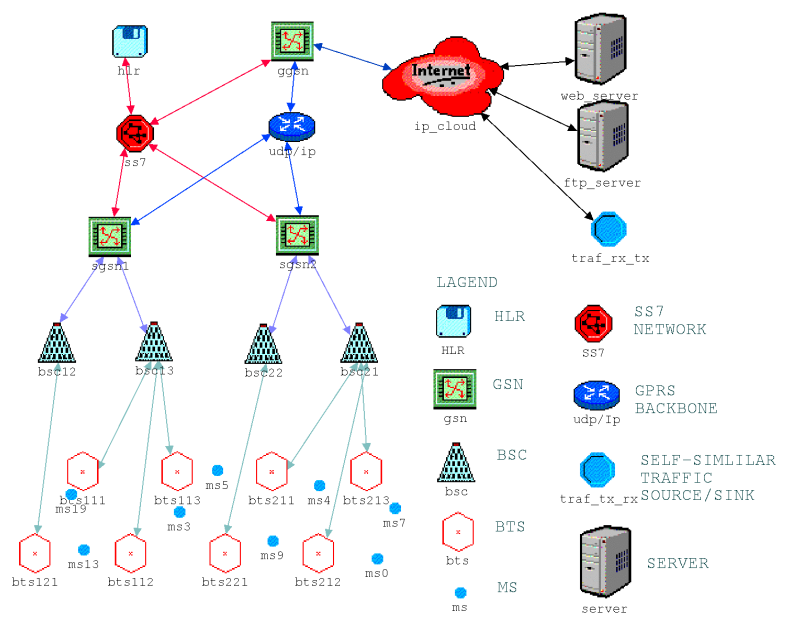

Figure 1: A top level view of OPNET GPRS model

\section{Simulation Parameters}

The assumptions and limitations of the GPRS simulation environment are as following. First, an ideal wireless interface was used. Hence, packets transmitted over the wireless interface encounter no bit error or loss. Congestion over the air interface was also not modelled. Secondly, GSNs are assumed to have an unlimited buffer size. As such, the only reason for packet loss and extra delay is merely due to interruption during routing area update processes. Thirdly, PDP context for each MS has been establish prior to any downlink packet delivery or paging. Unless otherwise stated, parameters used in simulations are as following,
Traffic load

Mean cell resident time

LLC mode

Wireless link speed

Wired link speed

Mean Internet Delay

Mean cross SS7 time

IP MTU

TCP MSS

Ready timer

Tunnel timer

Paging timer

Simulation time

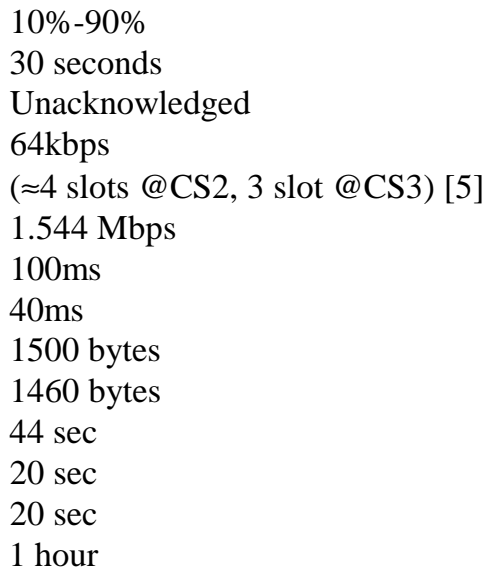

$20 \mathrm{sec}$

$20 \mathrm{sec}$

1 hour

Our simulation study were carried out on several performance measures, defined as following: 
1. Routing Area Update Delay - The time difference between MS starts sending routing area update request message to SGSN and SGSN finished receiving routing area update complete message from the same MS.

2. Packet Loss ratio - Ratio of total number of packet loss to the total number of packets transmitted.

3. Packet Loss per routing area update - The ratio of total number of packet loss to the total number of routing area update executed.

4. Throughput - The amount of data, bytes per second, forwarded to the application layer by TCP layer.

\section{Simulation Results}

Effect of Mobility. We first analysed the interruption of routing area update in packet delivery in term of routing area completion time and number of packet lost during routing area update. Figure 3 shows the differences of routing area completion time for two type of Routing Area Update when the network is $40 \%$ loaded. It can be seen that the average delay for inter-SGSN RAU (0.402 second) is much higher than that of intra-SGSN $(0.050$ second). Hence, it is expected that the interruption due to inter-SGSN RAU is higher, as evident in Figure 4. Figure 4 shows the average time a MS required to download a file of 2Mbytes while executing only either inter-SGSN RAUs or intra-SGSN RAUs.

Minimizing Effect of mobility by Queuing Algorithms. Next we simulated the performance under different service schemes, as described in section III, aiming at reducing the completion time of routing area update, and hence the disruption of packet delivery. We examine each measure as a function of traffic load in the GPRS network. Figure 5 and Figure 6 show that with FIFO scheme, the completion time for routing area update procedure increases exponentially with traffic load. The RAU completion time is reduced for Fair Queuing scheme. This is because a fairer share of resource has been allocated to processing each type of messages, namely MAP, LLC, and GTP. The delay for delivering MAP messages has been much reduced in this scheme. A MAP message is ensured to be served at each round. In Priority Queuing scheme, as we give priority to service mobility management-related messages, the completion time is kept constantly low though offered traffic increases. This is because under this scheme, regardless or the amount of traffic and length of the queue, the mobility management messages will always be at the top of the queue, and hence be served at the minimal delay.

Packet Loss Ratio. We have also ran a set of simulations for different cell resident times. Figure 7 shows the packet loss ratio due to mobility of MS. Again, Priority Queuing reveal the best performance, followed by Fair Queuing and then FIFO.
FTP Throughput. In another set of simulation, we investigated the performance of FTP and HTTP with different scheduling schemes. A FTP session is run to download a single large file. The Tahoe version of TCP was used and the receiver buffer size was set to 8760 bytes. The mean FTP throughput for as stationary MN was recorded for an unloaded GPRS network. The normalised throughputs on different traffic loads and the three service schemes are then recorded and depicted in Figure 8. We could see that the FTP throughput for prioritising mobility management messages is maintained constantly higher than that of Fair Queuing and FIFO.

\section{CONCLUSION}

We presented a simulation environment for performance analysis of packet delivery in GRPS with different parameter settings. The effects of mobility or Routing area update on packet data delivery in GPRS were analysed. The disruption on packet delivery during RAU, particularly during an inter-SGSN RAU could be very high as traffic load increases. A simple mechanism based on service scheduling was proposed and implemented to improve the performance of mobility management in GPRS by reducing the RAU completion time. Under fair queuing and priority based queuing, the delays in completing RAU are much lower as compared to first in first out. Hence, disruptions in data packet delivery are reduced.

\section{ACKNOWLEDGEMENT}

The work reported in this paper has formed part of the Networks and Services work area of the Core 2 Research Programme of the Virtual Centre of Excellence in Mobile \& Personal Communications, Mobile VCE, whose funding support is gratefully acknowledged. More detailed technical reports on this research are available to Industrial Members of MVCE.

\section{REFERENCES}

[1] ETSI, 2001, "Digital Cellular Telecommunications System (Phase 2+); General Packet Radio Service; Service Description; Stage 2",GSM 03.60.

[2] Kleinrock, L., 1976, "Queuing Systems Vol. 1", Wiley, New York, USA.

[3] www.opnet.com

[4] Ryu B et al., 2000, "Traffic Models for Internet Simulation”, ISCC, 200-206.

[5] ETSI, 2001, "Digital Cellular Telecommunications System (Phase 2+); General Packet Radio Service; Overall Description of the GPRS Radio Interface; Stage 2", GSM 03.64. 


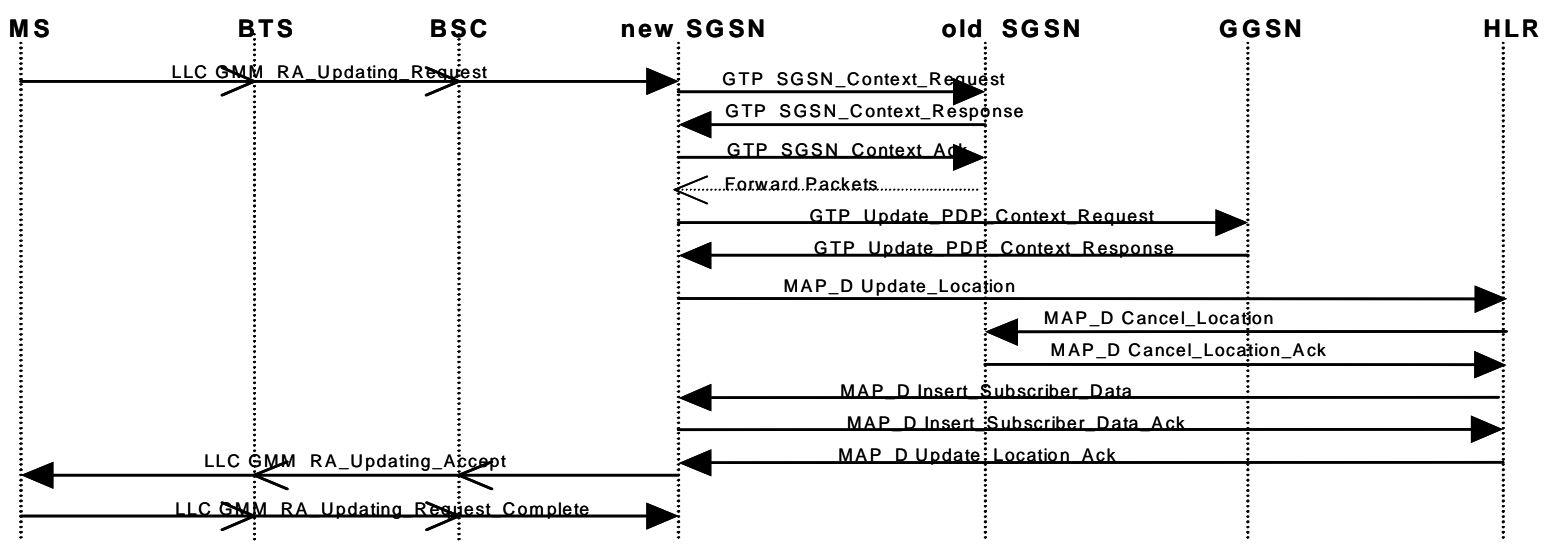

Figure 2: Inter-SGSN Routing Area Update Procedure

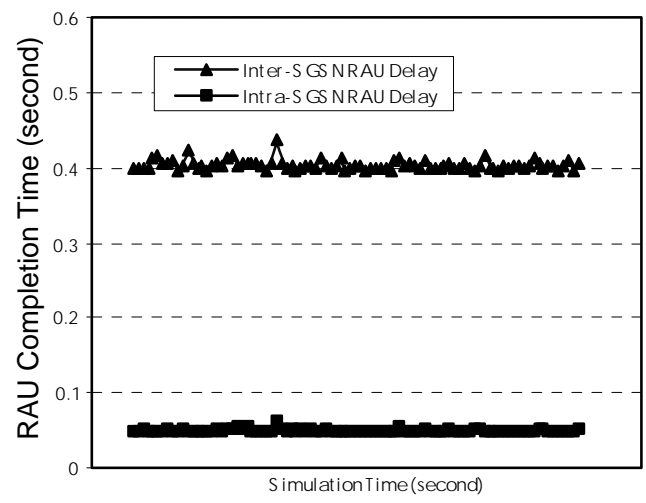

Figure 3: Average RAU Completion Time

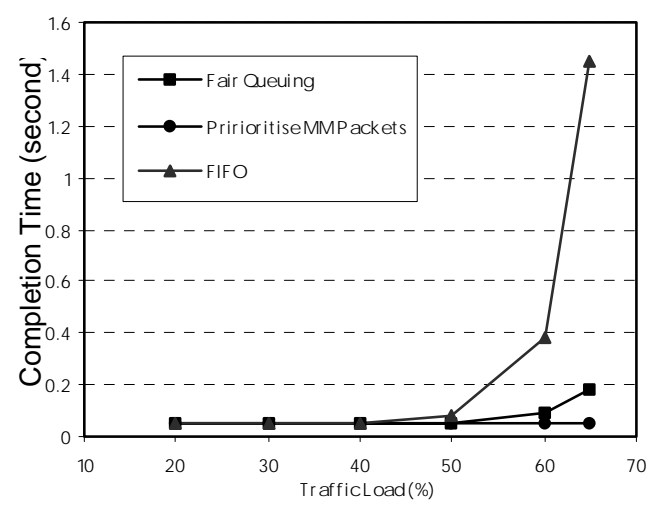

Figure 5: Intra-SGSN RAU Completion Time

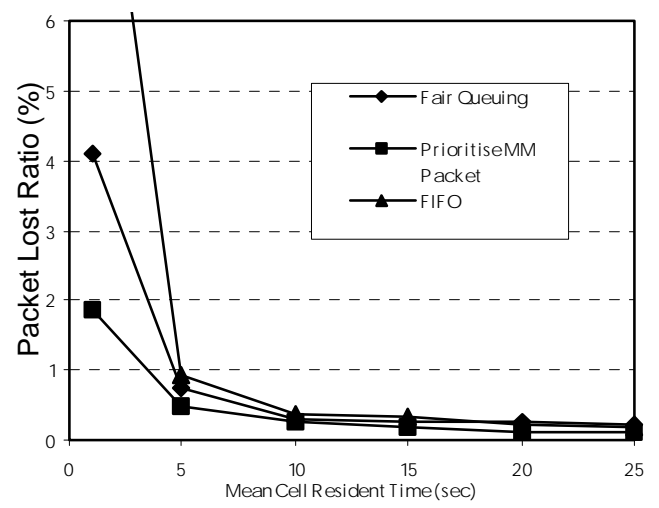

Figure 7: Packet Loss Ratio

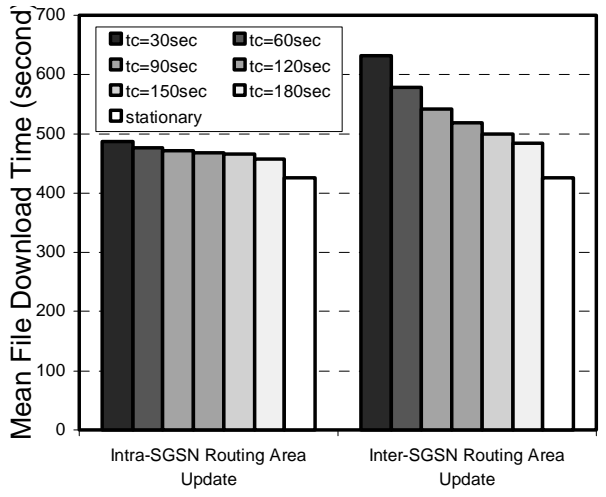

Figure 4: File download time for different types of RAU

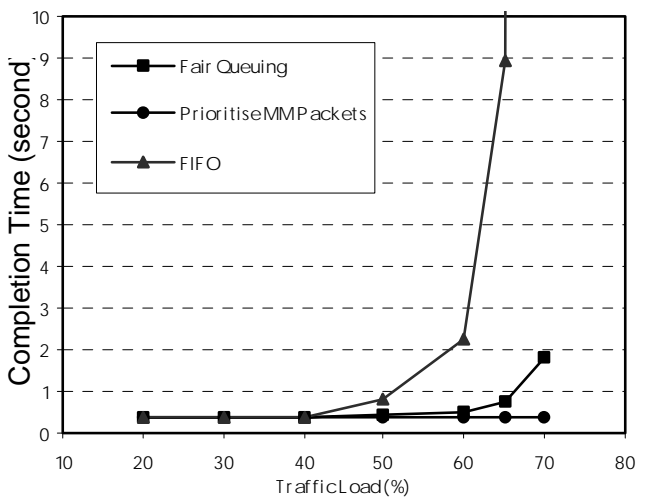

Figure 6: Inter-SGSN Routing Area Completion Time

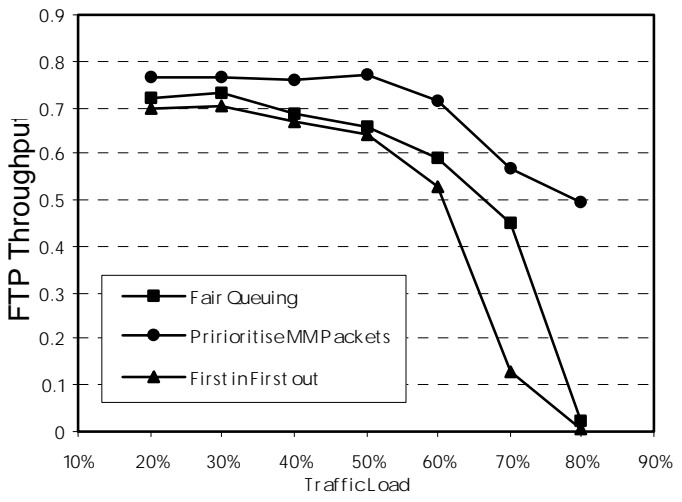

Figure 8: FTP throughput 